\title{
THE 3D QUASIGEOSTROPHIC FLUID DYNAMICS UNDER RANDOM FORCING ON BOUNDARY*
}

\author{
JINQIAO DUAN ${ }^{\dagger}$ AND BJÖRN SCHMALFUSS $\ddagger$
}

\begin{abstract}
The three-dimensional baroclinic quasigeostrophic flow model has been widely used to study basic mechanisms in oceanic flows and climate dynamics. In this paper, we consider this flow model under random wind forcing and time-periodic fluctuations on fluid boundary (the airsea interface). The time-periodic fluctuations are due to periodic rotation of the earth and thus periodic exposure of the earth to the solar radiation. After overcoming the difficulty due to the low regularity of an associated Ornstein-Uhlenbeck process, we establish the well-posedness of the baroclinic quasigeostrophic flow model in the state space. Then we demonstrate the existence of the random attractors, again in the state space. We also discuss the relevance of our result to climate modeling.
\end{abstract}

Key words. 3D Quasigeostrophic flows, wind forcing on boundary, stable stationary flows, random dynamical systems, random attractors

AMS subject classifications. Primary 60H25, 76D05, 47H10; Secondary 34D35, 86A05

\section{Introduction}

The quasigeostrophic equation (QGE) describes large scale geophysical flows. We consider this flow model for the ocean in a three-dimensional (3D) domain. This geophysical flow model has been formally derived as an approximation of the rotating three-dimensional primitive equations [21]. It is recently known that the threedimensional quasigeostrophic model is a valid approximation of the primitive equations in the limit of zero Rossby number [3, 10, 6]. Holm [16] has established the Hamiltonian formulation for the inviscid quasigeostrophic equation. We will consider the following version $[32,8]$ of the quasigeostrophic equation in terms of the stream function $\psi(x, y, z, t)$ :

$$
\begin{aligned}
\tilde{\Delta} \psi_{t}+J(\psi, \tilde{\Delta} \psi) & +\beta \psi_{x}=\nu \tilde{\Delta} \tilde{\Delta} \psi \\
\text { with } \quad \tilde{\Delta} \psi & =\psi_{x x}+\psi_{y y}+\frac{f_{0}^{2}}{N^{2}} \psi_{z z} .
\end{aligned}
$$

Here $x, y, z$ are Cartesian coordinates in zonal (east), meridional (north), vertical directions, respectively; $f_{0}+\beta y$ (with $f_{0}, \beta$ constants) is the Coriolis parameter; $N>0$ is the Brunt-Vaisala stratification frequency taking to be constant in this paper, and $\nu>0$ is viscosity. Moreover, $J(f, g)=f_{x} g_{y}-f_{y} g_{x}$ is the Jacobi operator, and potential vorticity is defined as $\tilde{\Delta} \psi+f_{0}+\beta y$. Note that $\tilde{\Delta} \psi$ can be regarded as a modified Laplacian operator where the coefficient in the vertical $z$ direction is adjusted due to the density stratification, and the coefficients in $x, y$ directions are constants due to the horizontal density homogeneity in the 3D quasigeostrophic flow model formulation. Bennett and Kloeden [7] have also used a similar modified Laplacian viscous term in a more complicated 3D quasigeostrophic flow model involving thermodynamics as well as hydrodynamics.

\footnotetext{
*Received: May 1, 2002; Accepted (in revised version): August 26, 2002.

${ }^{\dagger}$ Department of Applied Mathematics, Illinois Institute of Technology, Chicago, IL 60616, USA (duan@iit.edu).

$\ddagger$ Department of Applied Sciences, University of Technology and Applied Sciences, Geusaer Strasse D-06217, Merseburg, Germany (schmalfuss@in.fh-merseburg.de).
} 
Our aim is to study the potential vorticity evolution in an ocean under the influence of the atmosphere. Oceanic flows are affected (on the ocean surface) by the short time influence due to random weather variations which are usually called random wind forcing. This wind forcing is usually of shorter time scale than the response time scale of the large scale oceanic flows such as the quasigeostrophic flows. So we neglect the autocorrelation time of this fluctuating process as in [20]. We thus assume the noise is white in time. The random white-in-time noise is described as the generalized time-derivative of a Wiener process $W(x, y, t)$ with mean zero and covariance operator $Q$. Moreover, oceanic flows are also affected by climatic variations due to periodic rotation of the earth and thus periodic exposure of the earth to the solar radiation; see [22], Chapter 6 and [17], Chapter 11.

Since the exchange between the atmosphere and an ocean takes place at the surface of the ocean, we will consider the above quasigeostrophic equation with white noise Neumann boundary condition and time-periodic boundary condition on the top surface of the ocean [21]. Since there is no influence of the weather at the bottom of the ocean, we will impose homogeneous boundary condition there. The boundary conditions in horizontal directions are assumed to be periodic as in other recent works $[10,3]$. However, since we have a forth order differential operator at the right hand side of the quasigeostrophic equation, we need a second group of boundary conditions, say, for $\tilde{\Delta} \psi$, and these conditions are the ones used in [32], [8] to be specified below.

Due to the time-dependent random and time-periodic boundary conditions, we are dealing with a nonautonomous dynamical system with random influences. We will show how to find attractors for such a dynamical system. The existence and interpretation of climatic attractors have been controversial and have caused a lot of debate [19]. A low dimensional climatic attractor was regarded as an indication that the main feature of long-time climatic evolution may be viewed as the manifestation of a deterministic dynamics. Our result is about random attractors, and thus the long time regimes that such attractors may represent still carry the stochastic information of the geophysical flow system. Stochastically forced QGE has been used to investigate various mechanisms in geophysical flows [15, 18, 27, 4].

The plan for this paper is as follows. The QGE will be transformed into a random evolution equation with homogeneous and deterministic boundary conditions. We thus need an Ornstein-Uhlenbeck process fulfilling our dynamical random or timeperiodic boundary conditions. This transformation will be introduced in Section 2.

In Section 3 we investigate the coefficients of the transformed evolution equation and further obtain a global existence and uniqueness result and some regularity result. A difficult issue here is the low regularity of the above Ornstein-Uhlenbeck process.

In Section 4, we study the random dynamics of the transformed QGE. Based on the uniqueness result above, the transformed evolution equation generates a nonautonomous dynamical system. In addition, if we restrict this system to discrete time steps of the period of the periodic rotation of the earth, we obtain a random dynamical system. This random dynamical system has a random attractor. This result can be extended to the dynamical system on the real-time axis.

The Section 5 contains the proofs. Finally, we summarize our results in Section 6.

\section{3D Quasigeostrophic Flow Model}

Let $O=(0,2 \pi)^{3}$ be the cube which is a model for a part of the ocean. The differential operator $\tilde{\Delta}$ is defined by

$$
\tilde{\Delta}:=\partial_{x x}+\partial_{y y}+\left(F \partial_{z}\right)_{z}
$$


with $F:=\frac{f_{0}^{2}}{N^{2}}$. In the following, we investigate the 3D QGE flow model [21, 26, 32]:

$$
\tilde{\Delta} \psi_{t}+J(\psi, \tilde{\Delta} \psi)+\beta \psi_{x}=\nu \tilde{\Delta} \tilde{\Delta} \psi .
$$

We impose the following boundary conditions for this equation. Let $O_{,,, 0}, O_{r,, 2 \pi}, \cdots$ be the faces of the cube $O$, then we assume periodic boundary conditions in $x, y$ directions:

$$
\left.\psi\right|_{O_{0,,,}}=\left.\left.\psi\right|_{O_{2 \pi,,,}} \quad \psi_{x}\right|_{O_{0,,,}}=\left.\psi_{x}\right|_{O_{2 \pi, \cdot,}}
$$

and similar for the faces $O_{\cdot, 0,}, O_{., 2 \pi,}$ together with derivative in $y$ direction. With respect to the $z$ direction, $\psi$ fulfills homogeneous Neumann boundary conditions on top and bottom boundaries:

$$
\left.\psi_{z}\right|_{O,,, 0}=0,\left.\quad \psi_{z}\right|_{O,,, 2 \pi}=0 .
$$

Note that we could also consider Dirichlet boundary conditions on these faces. Moreover, $\tilde{\Delta} \psi$ is supposed to be periodic in $x, y$ directions:

$$
\left.\tilde{\Delta} \psi\right|_{O_{0,,},}=\left.\left.\tilde{\Delta} \psi\right|_{O_{2 \pi, \cdot,}} \quad \tilde{\Delta} \psi_{x}\right|_{O_{0, \cdots,}}=\left.\tilde{\Delta} \psi_{x}\right|_{O_{2 \pi, \cdot,}}
$$

and similar for the faces $O_{\cdot, 0,}, O_{, 2 \pi,}$, with derivative in $y$ direction. On the top of the ocean we impose white noise Neumann boundary conditions as in $[32,8,7]$, while on the bottom we define homogeneous Neumann conditions. Namely, we impose that

$$
\frac{\partial \tilde{\Delta} \psi}{\partial n}=\tilde{\Delta} \psi_{z}=0 \quad \text { on bottom boundary } O_{,,,}, 0
$$

and

$$
\frac{\partial \tilde{\Delta} \psi}{\partial n}=\tilde{\Delta} \psi_{z}=\dot{W}+u_{p} \sin (2 \pi t) \quad \text { on top boundary } O_{\cdot,, 2 \pi},
$$

where $\dot{W}(x, y, t)$ is a temporal white noise, $u_{p}(x, y)$ is the spatial profile for the timeperiodic fluctuations at the air-sea interface, and $n$ denotes the unit outer normal vector. We note that other boundary conditions may be considered for this flow model.

In addition, we impose the zero-mean condition

$$
\int_{O} \psi d O=0, \quad \text { and } \quad \int_{O} \tilde{\Delta} \psi d O=0 .
$$

We also assume an appropriate initial condition

$$
\psi(x, y, z, 0)=\psi_{0}(x, y, z) .
$$

Later on we will see that we can find an Ornstein-Uhlenbeck stochastic process $\eta$ satisfying the linear differential equation

$$
\eta_{t}=\nu \tilde{\Delta} \eta, \quad \eta(0)=\eta_{0}
$$

where $\eta$ fulfills the same (partially random) boundary conditions as $\tilde{\Delta} \psi$.

We now formally transform the QGE equation to a partial differential equation with random coefficients but with homogeneous and deterministic boundary conditions. 
To do so we introduce the solution operator $G$ of the following elliptic problem:

$$
\tilde{\Delta} \xi=f
$$

on $O$ with periodic boundary conditions in $x, y$ directions and homogeneous Neumann boundary conditions in $z$ direction. Here $f$ is an element in a Hilbert space to be specified below.

We now set

$$
u:=\tilde{\Delta} \psi-\eta
$$

and rewrite equation $(2.1)$ as

$$
\begin{aligned}
u_{t}-\nu \tilde{\Delta} u & +J(G(u), u)+\beta G(u)_{x}= \\
= & -J(G(\eta), u)-J(G(u), \eta)-J(G(\eta), \eta)-\beta G(\eta)_{x}, \quad u(0)=u_{0}
\end{aligned}
$$

where $u$ satisfies homogeneous Neumann boundary conditions in $z$ direction and periodic boundary conditions in $x, y$ directions. Equation (2.6) will serve as a main model for our investigation in the rest of this paper.

\section{Well-Posedness of 3D Quasigeostrophic Flows}

In this section we consider the well-posedness of the transformed QGE (2.6). We treat equation (2.6) as an evolution equation on a rigged space $V \subset H \subset V^{\prime}$. Since this equation is given with respect to periodic boundary conditions in horizontal directions and homogeneous Neumann conditions in the vertical direction, the space Hilbert $H$ is defined to be

$$
\dot{L}_{2}(O)=\left\{u \in L_{2}(O), \int_{O} u d O=0\right\}
$$

The usual inner product on $H$ is denoted by $(\cdot, \cdot)$. Let $H^{k}, k \in \mathbb{N}$ be the usual Sobolev space consisting of functions with square integrable derivative up to $k$-th order. If $k$ is not an integer, these spaces are the (generalized) Sobolev spaces. We define $V:=H^{1} \cap H$ with

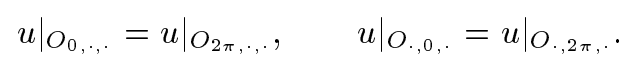

Note that for functions in $H^{1}$, the trace on the boundary is well defined. This set $V$ will be equipped with the usual $H^{1}$ inner product denoted by $(\cdot, \cdot)_{V}$. Let $A$ be a linear bounded operator

$$
A: V \rightarrow V^{\prime}
$$

which is the usual operator stemming from the positive bilinear form $a(u, v)$ defined by $-\tilde{\Delta}$ with periodic boundary conditions in $x, y$ directions and homogeneous Neumann boundary condition in $z$ direction. Note that $-\tilde{\Delta}$ is symmetric with respect to these boundary conditions. We have that

$$
\langle A u, u\rangle=\|u\|_{V}^{2} .
$$

$\langle\cdot, \cdot\rangle$ denotes the dual pairing between $V=D\left(A^{\frac{1}{2}}\right)$ and $V^{\prime}$. The operator $A$ is an isomorphism from $V$ to $V^{\prime}$. Since $V$ is compactly embedded in $H$, we can define $G(f)=A^{-1} f$, which is a continuous operator from $H$ to $D(A)$ or more generally from 
$D\left(A^{s}\right) \subset H^{2 s}$ to $D\left(A^{s+1}\right), s \in \mathbb{R}$. For the definition of the spaces $D\left(A^{s}\right)$ and their norms $\|\cdot\|_{D\left(A^{s}\right)}$ see Temam [31], Section II.2. In particular, for $f \in H$ the function $G(f)$ is periodic in $x, y$ directions and satisfies homogeneous Neumann condition in $z$ direction. We will stress that the norm of $H^{2 s}, s \geq 0$ restricted to $D(A)^{s}$ is equivalent to $\|\cdot\|_{D\left(A^{s}\right)}$.

On $H$, the operator $A$ has the spectrum $0<\lambda_{1} \leq \lambda_{2} \leq \ldots$. The associated eigenfunctions $e_{1}, e_{2}, \cdots$ form a complete orthonormal base in $H$.

The embedding constant between $V$ and $H$ is given by $\lambda_{1}$ in the Poincare inequality:

$$
\lambda_{1}\|u\|_{H}^{2} \leq\|u\|_{V}^{2}
$$

We now investigate the properties of the operator $J$, which defines the nonlinearity of $(2.6)$.

Lemma 3.1. Suppose that $m_{1}, m_{2}, m_{3}$ are three nonnegative numbers less than $\frac{3}{2}$ with

$$
\sum_{i=1}^{3} m_{i} \geq \frac{3}{2}
$$

Then there exists a constant $c>0$ such that for $u \in H^{m_{1}+1}, v \in H^{m_{2}+1}$ and $w \in H^{m_{3}}$

$$
|\langle J(u, v), w\rangle| \leq c\|u\|_{H^{m_{1}+1}}\|v\|_{H^{m_{2}+1}}\|w\|_{H^{m_{3}}} .
$$

Proof. The Hölder inequality implies

$$
|\langle J(u, v), w\rangle| \leq\|\nabla u\|_{L_{q_{1}}}\|\nabla v\|_{L_{q_{2}}}\|w\|_{L_{q_{3}}}, \quad \frac{1}{q_{1}}+\frac{1}{q_{2}}+\frac{1}{q_{3}} \leq 1 .
$$

The embedding property $H^{m} \subset L_{q}(O)$ where $\frac{1}{q}=\frac{1}{2}-\frac{m}{3}$ for $q>1, m \geq 0, m \neq \frac{3}{2}$ gives the conclusion. For the idea of the proof see Temam [30], Section 2.3.

REMARK 3.2. Suppose two of the $m_{i}$ 's in the last lemma, say $m_{2}, m_{3}$, have the value zero. Then if $m_{1}$ is chosen bigger than $\frac{3}{2}$, the conclusion of the last lemma remains true. This follows if we apply at first the Sobolev lemma and then the CauchySchwarz inequality. From Lemma 3.1 we can derive some a priori estimates for the nonlinearity of (2.6).

Corollary 3.3. Suppose that $u \in D\left(A^{\alpha}\right), \alpha>\frac{3}{4}$, and $v, w \in V$. Then for some $c$

$$
|\langle J(u, v), w\rangle| \leq c\|u\|_{D\left(A^{\alpha}\right)}\|v\|_{V}\|w\|_{V},
$$

and hence for $u \in H, v, w \in V$

$$
|\langle J(G(u), v), w\rangle| \leq c\|u\|_{H}\|v\|_{V}\|w\|_{V} .
$$

Suppose now that $u \in D\left(A^{\alpha}\right), \alpha>\frac{5}{4}$. Then

$$
|\langle J(u, v), w\rangle| \leq c\|u\|_{D\left(A^{\alpha}\right)}\|v\|_{V}\|w\|_{H} \quad \text { for } \quad v \in V, w \in H
$$


and hence for $u \in D\left(A^{\alpha}\right), \alpha>\frac{1}{4}$

$$
|\langle J(G(u), v), w\rangle| \leq c\|u\|_{D\left(A^{\alpha}\right)}\|v\|_{V}\|w\|_{H} \quad \text { for } \quad v \in V, w \in H .
$$

REMARK 3.4. Since $\mathcal{O}$ is a cubic domain, the eigenfunctions of $-\tilde{\Delta}$ are defined by $c(\vec{k}) \exp (i \vec{k} \cdot(x, y, z))$ where $\vec{k}$ is in an appropriate integer grid of $\mathbb{R}^{3}$. Indeed, the cos-part of the Neumann ansatz in $z$ direction can be expressed by such terms. Hence we can generalize the conclusion of Lemma 3.1 to the case when $m_{i} \in \mathbb{R}$, and the following conditions are satisfied:

$$
m_{i}+m_{j}>0 \text { for } i \neq j
$$

instead of $m_{i} \geq 0$. For the proof, we refer to Fursikov [13], Chapter 3, Section 4, Lemma 4.4.

LEMma 3.5. There exists a constant $c$ such that for any $\eta \in D\left(A^{\gamma}\right), 0<\gamma<1 / 4$ and $u \in H^{1}$, we have

$$
|\langle J(G(u), \eta), u\rangle| \leq c\|u\|_{0}^{\frac{1}{2}}\|\eta\|_{D\left(A^{\gamma}\right)}\|u\|_{1}^{\frac{3}{2}} .
$$

Proof. For $u \in D\left(A^{\frac{1}{2}}\right)$ we have

$$
\|G(u)\|_{2+1 / 2} \leq c\|u\|_{\frac{1}{2}} \leq\|u\|_{0}^{\frac{1}{2}}\|u\|_{1}^{\frac{1}{2}}
$$

which follows by an interpolation inequality, see Temam [31]. By Lemma 3.1 and Remark 3.4 with $m_{1}=\frac{3}{2}, m_{2}=-1+2 \gamma, m_{3}=1$, we finally obtain

$$
|\langle J(G(u), \eta), u\rangle| \leq c\|G(u)\|_{1+3 / 2}\|\eta\|_{1-(1-2 \gamma)}\|u\|_{1} .
$$

Also on account of Lemma 3.1 we obtain the following algebraic properties of $J$.

Lemma 3.6. If $u, v, w \in V$ then we have

$$
\langle J(u, v), w\rangle=-\langle J(u, w), v\rangle .
$$

Hence we have $\langle J(u, v), v\rangle=0$.

Proof. We choose $u$ from a set of sufficiently smooth functions which is dense in $D(A)$. Integration by parts yields

$$
\begin{aligned}
& \int_{0}^{2 \pi} \int_{O,,, z} u_{x} v_{y} w d x d y d z-\int_{0}^{2 \pi} \int_{O,,, z} u_{y} v_{x} w d x d y d z \\
& =-\int_{0}^{2 \pi} \int_{O_{,,,, z}} u_{x y} v w d x d y d z+\int_{0}^{2 \pi} \int_{O_{,,,, z}} u_{y x} v w d x d y d z \\
& -\int_{0}^{2 \pi} \int_{O, \ldots, z} u_{x} v w_{y} d x d y d z+\int_{0}^{2 \pi} \int_{O, \ldots, z} u_{y} v w_{x} d x d y d z \\
& +\left.\int_{0}^{2 \pi} \int_{0}^{2 \pi} u_{x} v w\right|_{y=0} ^{y=2 \pi} d x d z-\left.\int_{0}^{2 \pi} \int_{0}^{2 \pi} u_{y} v w\right|_{x=0} ^{x=2 \pi} d y d z .
\end{aligned}
$$


Note that the two boundary terms are zero. Indeed, since $u(x, 0, z)=u(x, 2 \pi, z)$, we know that $u_{x}(x, 0, z)=u_{x}(x, 2 \pi, z)$, and since $v, w$ are $2 \pi$-periodic with respect to $x$. By the smoothness assumption we can suppose that the derivatives on the boundary are well defined. Thus the first boundary term is zero. Similarly, the second boundary term is also zero. By the continuity of $J$ (see Corollary 3.3) we can extend the relation to $V \times V \times V$. The second claim is due to antisymmetric property of the first claim of this corollary.

The following lemma will be used to obtain the continuity of the solution operator of $(2.6)$.

LEMma 3.7. There exists a constant $c>0$ such that for $u_{1}, u_{2} \in V$

$$
\left|\left\langle J\left(G\left(u_{1}\right), u_{1}\right)-J\left(G\left(u_{2}\right), u_{2}\right), u_{1}-u_{2}\right\rangle\right| \leq c\left\|u_{1}-u_{2}\right\|_{V}\left\|u_{1}-u_{2}\right\|_{H}\left\|u_{1}\right\|_{V} .
$$

Proof. By Lemma 3.6 the left-hand side in the above expression is equal to

$$
\begin{aligned}
\mid\left\langle J\left(G\left(u_{1}\right), u_{1}\right), u_{2}\right\rangle & +\left\langle J\left(G\left(u_{2}\right), u_{2}\right), u_{1}\right\rangle \mid \\
& =\left|\left\langle J\left(G\left(u_{1}\right), u_{1}\right), u_{2}-u_{1}\right\rangle-\left\langle J\left(G\left(u_{2}\right), u_{1}\right), u_{2}-u_{1}\right\rangle\right| \\
& =\left|\left\langle J\left(G\left(u_{1}\right)-G\left(u_{2}\right), u_{1}\right), u_{1}-u_{2}\right\rangle\right| .
\end{aligned}
$$

Thus Corollary 3.3 gives the conclusion.

In order to write equation (2.6) as an evolution equation on the rigged space $V \subset H \subset V^{\prime}$ introduced at the beginning of this section, we need to check the properties of coefficients in equation (2.6). Due to Corollary 3.3, we have a bilinear continuous operator

$$
B(\cdot, \cdot): V \times V \rightarrow H, \quad B(u, v):=J(G(u), v) .
$$

In addition, we have a time-dependent linear continuous operator $C(t, \cdot): V \rightarrow V^{\prime}$

$$
C(t, u):=J(G(u), \eta(t))+J(G(\eta(t)), u)
$$

where the Ornstein-Uhlenbeck process $\eta(t)$ defined in $(2.4)$ is in $C\left([0, \infty) ; D\left(A^{\gamma}\right)\right), 0<$ $\gamma<1 / 4$ and is periodic in $x, y$ directions. Note that $G(\eta(t)) \in D\left(A^{1+\gamma}\right)$. The existence and properties of $\eta(t)$ will be proved in the next section.

Lemma 3.8. Suppose that $\eta(\cdot) \in C\left([0, \infty) ; D\left(A^{\gamma}\right)\right), 0<\gamma<1 / 4$. Then we have for $t \geq 0$ :

$$
\|C(t, \cdot)\|_{\mathcal{L}\left(V, V^{\prime}\right)} \leq c\|\eta(t)\|_{D\left(A^{\gamma}\right)} .
$$

Indeed, because $G(\eta) \in D\left(A^{1+\gamma}\right)$ for any $t \geq 0$ we obtain the conclusion by Corollary 3.3 and Remark 3.4.

We now investigate the last linear operator appearing in (2.6) which is defined by

$$
D(\cdot)=\beta G(\cdot)_{x}: V \rightarrow H^{2}, \quad D(\cdot): D\left(A^{\gamma}\right) \rightarrow H^{1+2 \gamma} .
$$

Lemma 3.9. For $u \in V$ we have

$$
(D(u), u)=0 .
$$


Proof. Denoting $G(u) \in D(A)$ by $\xi$ we have

$$
\begin{aligned}
\beta^{-1}(D(u), u) & =\left(\xi_{x}, \tilde{\Delta} \xi\right)=\frac{1}{2}\left(\int_{O}\left(\xi_{x}^{2}\right)_{x} d x d y d z-\left(\xi_{y}^{2}\right)_{x} d x d y d z\right. \\
& \left.-\int_{0}^{2 \pi} \int_{0}^{2 \pi} F \int_{0}^{2 \pi}\left(\xi_{z}^{2}\right)_{x} d x d y d z\right)
\end{aligned}
$$

via the integration by parts. The second term under the integral generates the boundary term

$$
\left.\frac{1}{2} \int_{0}^{2 \pi} \int_{0}^{2 \pi} \xi_{x} \xi_{y}\right|_{y=0} ^{y=2 \pi} d x d z .
$$

Indeed, for sufficiently smooth $\xi$ from a dense set in $V$ we have $\xi(x, 0, z)=\xi(x, 2 \pi, z)$, and thus $\xi_{x}(x, 0, z)=\xi_{x}(x, 2 \pi, z)$. It follows from the periodicity in $y$ we have $\xi_{y}(x, 0, z)=\xi_{y}(x, 2 \pi, z)$ such that this boundary term is zero.

For the last term the following boundary term appears

$$
\left.\frac{1}{2} \int_{0}^{2 \pi} \int_{0}^{2 \pi} F \xi_{x} \xi_{z}\right|_{z=0} ^{z=2 \pi} d x d y
$$

which is zero by the homogeneous Neumann boundary conditions.

Integration with respect to $x$ and using the periodicity as in the proof of Lemma 3.6 , we get the conclusion.

We can now formulate the evolution equation (2.6) on $V \subset H \subset V^{\prime}$

$$
u_{t}+\nu A u+B(u, u)+C(t, u)+D(u)=f(t), \quad u(0)=u_{0} \in H
$$

for $f(t) \in V^{\prime}, f(t)=-J(G(\eta(t)), \eta(t))-\beta G(\eta(t))_{x}$ which is contained in $C\left([0, \infty) ; V^{\prime}\right)$. This regularity conclusion follows straightforwardly from Remark 3.4 for $0<\gamma<1 / 4$ sufficiently large.

Apart from the linear operators $C(t)$ and $D$, equation (3.1) has the form of equations of 2D Navier-Stokes type; see Temam [29], Chapter 3, for which we have existence and uniqueness, via the Galerkin approximation. Here $2 D$ means that the conclusion of Lemma 3.7 is fulfilled which is responsible for a uniqueness theorem. By the regularity properties of the operators $B, C(t)$ and $D$, the same method for the $2 \mathrm{D}$ Navier-Stokes equations ensures existence and uniqueness for (3.1). Thus we get the following main result in this section about the well-posedness for 3D quasigeostrophic flows under random wind forcing on ocean surface.

THEOREM 3.10. (Well-posedness) Suppose that the stochastic process $\eta(t)$ defined in (2.4) is in $C\left([0, \infty) ; D\left(A^{\gamma}\right)\right)$. Then for any initial condition $x \in H$, the $3 D$ quasigeostrophic flow model (3.1) or (2.6) has a unique (weak) solution $u(t) \in L_{2, l o c}(0, \infty ; V)$ $\cap C([0, \infty) ; H)$.

In the next section, we will show that the Ornstein-Uhlenbeck stochastic process $\eta(t)$ defined in (2.4) indeed has the required regularity.

We will study the random dynamics of 3D quasigeostrophic flow model in the next section. We thus need the some solution properties formulated in the following lemmas. 
Lemma 3.11. Let $u(t)$ be the solution of (3.1) for $u_{0} \in H$. Then the mapping

$$
H \ni u_{0} \rightarrow u(t) \in H
$$

is continuous for any $t \geq 0$.

Proof. Let $u_{1}(t), u_{2}(t)$ be two solutions for initial conditions $u_{01}, u_{02} \in H$. On account of Lemma 3.7, 3.9, 3.5, 3.8 and the Young inequality we have

$$
\begin{aligned}
\frac{d}{d t}\left\|u_{1}-u_{2}\right\|_{H}^{2} & +2 \nu\left\|u_{1}-u_{2}\right\|_{V}^{2} \\
& \leq 2\left|\left\langle B\left(u_{1}\right)-B\left(u_{2}\right), u_{1}-u_{2}\right\rangle\right|+2\left|\left\langle C(t)\left(u_{1}-u_{2}\right), u_{1}-u_{2}\right\rangle\right| \\
& \leq \nu\left\|u_{1}-u_{2}\right\|_{V}^{2}+c\left\|u_{1}\right\|_{V}^{2}\left\|u_{1}-u_{2}\right\|_{H}^{2} \\
& +c\|\eta(t)\|_{D\left(A^{\gamma}\right)}^{4}\left\|u_{1}-u_{2}\right\|_{H}^{2}+\nu\left\|u_{1}-u_{2}\right\|_{V}^{2}
\end{aligned}
$$

for an appropriate constant $c>0$. Now the Gronwall Lemma gives the conclusion.

Lemma 3.12. For any $t \in(0,1]$ there exists a function $c(t, \cdot, \cdot)$ bounded on bounded sets such that

$$
\|u(t)\|_{\gamma}^{2} \leq c\left(t,\left\|u_{0}\right\|_{H}, \sup _{t \in[0,1]}\|\eta(t)\|_{D\left(A^{\gamma}\right)}\right)
$$

Proof. We show that $t\|u(t)\|_{\gamma}^{2}$ is bounded for bounded $u_{0} \in H$. We have

$$
\frac{d}{d t}\left(t\|u(t)\|_{\gamma}^{2}\right)=\|u(t)\|_{\gamma}^{2}+2 t\left\langle\frac{d}{d t} u, A^{\gamma} u\right\rangle
$$

The second expression can be rewritten as

$$
\begin{aligned}
-2 t \nu\|u\|_{D\left(A^{\frac{1+\gamma}{2}}\right)}^{2} & -2 t\left\langle J(G(u), u), A^{\gamma} u\right\rangle-2 t\left\langle J(G(u), \eta), A^{\gamma} u\right\rangle \\
& -2 t\left\langle J(G(\eta), u), A^{\gamma} u\right\rangle-2 t\left\langle J(G(\eta), \eta), A^{\gamma} u\right\rangle \\
& -2 t\left\langle D(u), A^{\gamma} u\right\rangle-2 t\left\langle D(\eta), A^{\gamma} u\right\rangle,
\end{aligned}
$$

where the third and fifth terms can be estimated by Corollary 3.3, Remark 3.4 for sufficiently large $\gamma<1 / 4$

$$
\begin{aligned}
2 t\left|\left\langle J(G(u), u), A^{\gamma} u\right\rangle\right| & \leq c t \sup _{t \in[0,1]}\|u(t)\|_{H}\|u\|_{1}\|u\|_{D\left(A^{\frac{1+\gamma}{2}}\right)} \\
& \leq \frac{\nu}{4} t\|u\|_{D\left(A^{\frac{1+\gamma}{2}}\right)}^{2}+c t \sup _{t \in[0,1]}\|u(t)\|_{H}^{2}\|u\|_{1}^{2} \\
2 t\left|\left\langle J(G(\eta), \eta), A^{\gamma} u\right\rangle\right| & \leq 2 t\|G(\eta)\|_{1+1+2 \gamma}\|\eta\|_{2 \gamma}\|u\|_{D\left(A^{\frac{1+2 \gamma-\gamma}{2}}\right)} \\
& \leq \frac{\nu}{4} t\|u\|_{D\left(A^{\frac{1+\gamma}{2}}\right)}^{2}+c t\|\eta\|_{2 \gamma}^{4} .
\end{aligned}
$$

The other terms can be estimated similarly. Hence $t\|u(t)\|_{\gamma}^{2}$ is bounded by an expression depending on $\left\|u_{0}\right\|_{H}$, $\sup _{t \in[0,1]}\|\eta(t)\|_{D\left(A^{\gamma}\right)}$ and the data of the problem. By the energy inequality (see $(5.2),(5.3)$, below) we are able to estimate $\int_{0}^{t}\|u(\tau)\|_{V}^{2} d \tau$ by these expressions. However, the usual way to calculate these expressions to ensure that $A^{\frac{1+\gamma}{2}}$ is well defined is to consider at first the Galerkin approximations $u_{m}$ and then letting $m \rightarrow \infty$ such that $u_{m}$ tends to a solution $u$ of (3.1). 


\section{Random Dynamical System of 3D Quasigeostrophic Flows}

In this section we study the dynamical behavior of QGE (3.1). In the following we are going to describe the background perturbations defined on the top boundary of $O$ which will influence the dynamical system generated by (3.1); see the boundary condition on the air-sea interface (2.3). We will have two different influences. The first perturbation is white noise which models the weather or the small scale impact of the atmospheric motion through wind forcing on the surface. The other one is a periodic motion which serves as a model for the impact due to periodic rotation of the earth and thus periodic exposure of the earth to the solar radiation; see [22], Chapter 6 and [17], Chapter 11.

In the first part of this section we are going to explain a dynamical model of the boundary conditions.

We consider the elliptic differential equation

$$
\tilde{\Delta} u=0, \quad \frac{\partial u}{\partial n}=0 \text { on } O_{\cdot,,, 0} \quad \frac{\partial u}{\partial n}=g \in H^{\frac{1}{2}}\left(O_{,,,, 2 \pi}\right) \cap \dot{L}_{2}\left(O_{\cdot,,, 2 \pi}\right) .
$$

On the other two pairs of faces we have periodic boundary conditions. This equation has a unique solution; see Egorov and Shubin [9], page 130f. The solution operator is a linear continuous operator with the image in $H^{2}$. We denote this operator by $\tilde{G}(f)$ and $U:=H^{\frac{1}{2}}\left(O_{,,, 2 \pi}\right) \cap \dot{L}_{2}\left(O_{,,, 2 \pi}\right)$ denotes a usual boundary space. Suppose that $g=0$, then the above problem generates the linear operator $A: V \rightarrow V^{\prime}$,

$$
V=\left\{u \in H^{1} \cap H: u(0, \cdot, \cdot)=u(2 \pi, \cdot, \cdot), u(\cdot, 0, \cdot)=u(\cdot, 2 \pi, \cdot)\right\},
$$

introduced in the last section. In addition, $-\nu A$ is the generator of a $C_{0}$-semigroup on $H$. This semigroup is exponentially stable: there exists $C, \lambda_{1}>0$ with

$$
\|S(t)\|_{\mathcal{L}(H, H)}^{2} \leq C e^{-\nu \lambda_{1} t} \quad \text { for } \quad t \geq 0 .
$$

Since $A$ is symmetric and positive, $\lambda_{1}>0$ is the smallest eigenvalue of $A$. We have $H^{2} \subset D\left(A^{\rho}\right), 0 \leq \rho<3 / 4$. Indeed, for such a $\rho$ no boundary conditions are fixed for the Neumann problem; see Da Prato and Zabczyk [23], Page 401 (A59). It follows that $\tilde{G}$ maps $U$ into $D\left(A^{\rho}\right)$ continuously.

Now we consider the random part of the boundary conditions. Let $W$ be a continuous temporal Wiener process with values in a Hilbert space $U=H^{\frac{1}{2}}\left(O_{,,, 2 \pi}\right) \cap$ $\dot{L}_{2}(O,,, 2 \pi)$. This Wiener process is defined for positive and negative times; see Arnold [1], page 547. The covariance operator of $W$ is denoted by $Q$, which is a positive symmetric linear operator on $U$ with finite trace. The dynamics of $W$ is given by the metric dynamical system consisting of a probability space $(\Omega, \mathcal{F}, \mathbb{P})$ and a flow $\theta$, $(\Omega, \mathcal{F}, \mathbb{P}, \theta)$ where $\mathbb{P}$ is the Wiener measure with covariance $Q$ and $\theta=\left(\theta_{t}\right)_{t \in \mathbb{R}}$ is the flow of the Wiener shift:

$$
W\left(\cdot, \theta_{t} \omega\right)=W(\cdot+t, \omega)-W(t, \omega) \quad \text { for } t \in \mathbb{R} .
$$

The mapping $\theta$ is $(\mathcal{B}(\mathbb{R}) \otimes \mathcal{F}, \mathcal{F})$-measurable and fulfills the property

$$
\theta_{t+\tau}=\theta_{t} \circ \theta_{\tau}, \quad t, \tau \in \mathbb{R} .
$$

For instance, we can choose $\Omega$ to be the set of continuous functions $C_{0}(\mathbb{R}, U)$ which are zero, at zero and for $\mathcal{F}$ we choose the Borel- $\sigma$-algebra of $C_{0}(\mathbb{R}, U)$. Note that $\mathbb{P}$ is ergodic with respect to $\theta$. Later on we have to restrict this metric dynamical system to fix particular dynamical properties. 
We now show the existence of a solution of (2.4) satisfying particular properties. At first we show that the following problem has a solution:

$$
\eta_{1 t}=\nu \tilde{\Delta} \eta_{1}, \quad \frac{\partial \eta_{1}}{\partial n}=0, \quad \text { on } \quad O_{\cdot,,, 0}, \quad \frac{\partial \eta_{1}}{\partial n}=\dot{W}(t), \quad \text { on } \quad O_{\cdot,,, 2 \pi}
$$

under periodic boundary conditions with respect to the other faces of the cube $O$ and a initial condition at time $t=0$. This solution will serve as a process which compensates the nonhomogeneous boundary conditions in (2.6). A similar problem has been considered in Da Prato and Zabczyk [25], Chapter 13 or [24].

Theorem 4.1. Suppose the covariance operator $Q$ of the Brownian motion $W$ is a linear symmetric operator on $U$ with finite trace. Then there exists a random variable $\omega \rightarrow \eta_{1}(\cdot, \omega) \in C\left([0,1] ; D\left(A^{\gamma}\right)\right), \gamma \in(0,1 / 4)$ defined on a $\left\{\theta_{j}\right\}_{j \in \mathbb{Z}}$-invariant subset in $\Omega$ such that

$$
\mathbb{E} \sup _{t \in[0,1]}\left\|\eta_{1}(t, \omega)\right\|_{D\left(A^{\gamma}\right)}^{2} \leq C\left(\operatorname{tr}_{U} Q\right)<\infty .
$$

This $\eta_{1}(t)$ is a solution of (4.2) with the random boundary condition satisfied. In addition, we have for $k \in \mathbb{N}$ :

$$
\eta_{1}\left(\cdot, \theta_{k} \omega\right)=S(k) \eta_{1}(\cdot, \omega)+A \int_{0}^{k+\cdot} S(k+\cdot-\tau) \tilde{G} d W(\omega, \tau) \quad \text { on } \quad[0,1] .
$$

Proof. Let $\gamma \in(0,1 / 4), \mu>1 / 2$ such that $\gamma+\mu \in(1 / 2,3 / 4)$. Then we have for sufficiently small $\varepsilon>0$ and $g \in D\left(A^{\rho}\right)$ for any $\rho<3 / 4$ and an appropriate $c$ :

$$
\begin{aligned}
\int_{0}^{1} \tau^{-\varepsilon}\|A S(\tau) g\|_{D\left(A^{\gamma}\right)}^{2} d \tau & =\int_{0}^{1} \tau^{-\varepsilon}\left\|A^{\gamma} A S(\tau) g\right\|_{H}^{2} d \tau \\
& \leq \int_{0}^{1} \frac{c}{\tau^{2-2 \mu+\varepsilon}}\|g\|_{D\left(A^{\gamma+\mu}\right)}^{2} d \tau<\infty .
\end{aligned}
$$

We have used a standard property for $C_{0}$-semigroups; see the formula (A32) in Da Prato and Zabczyk [23].

Let $g=g_{i}:=\tilde{G} Q^{\frac{1}{2}} e_{i} \in D\left(A^{\rho}\right), \rho<3 / 4$ where $\left\{e_{i}\right\}_{i \in \mathbb{N}}$ is a complete orthonormal system in $U$. Then since $Q$ is of trace class, and $\tilde{G}$ is continuous we have

$$
\int_{0}^{1} \tau^{-\varepsilon}\left\|A S(\tau) \tilde{G} Q^{\frac{1}{2}}\right\|_{\mathcal{L}_{2}\left(U, D\left(A^{\gamma}\right)\right)}^{2} d \tau<\infty
$$

where $\mathcal{L}_{2}$ denotes the space of Hilbert-Schmidt operators. Due to the formula (13.2.14) in Da Prato and Zabczyk [25], we know that the continuous Ornstein-Uhlenbeck process

$$
(t, \omega) \rightarrow A \int_{0}^{t} S(t-\tau) d \tilde{G} W(\omega, \tau) \in D\left(A^{\gamma}\right)
$$

solves (4.2) with initial condition zero.

Since $S(t)$ is exponentially stable, we conclude that

$$
\eta_{1}(t, \omega)=A \int_{0}^{t} S(t-\tau) d \tilde{G} W(\omega, \tau)+\sum_{j=0}^{\infty} S(j) A \int_{-1}^{0} S(-\tau) d \tilde{G} W\left(\theta_{-j} \omega, \tau\right)
$$


is well defined on a set $\Omega_{0}$ of measure one in $D\left(A^{\gamma}\right)$ for $t \in[0,1]$ such that

$$
\mathbb{E} \sup _{t \in[0,1]}\left\|\eta_{1}(t)\right\|_{D\left(A^{\gamma}\right)}^{2} \leq C\left(\operatorname{tr}_{U} Q\right)<\infty .
$$

Hence, $\eta_{1}(\cdot, \omega)$ is well defined for any $\omega \in \bigcap_{\mathbb{Z}} \theta_{j} \Omega_{0}$ which is a $\left\{\theta_{j}\right\}_{j \in \mathbb{Z}}$-invariant set of full measure. The formula (4.3) follows from the definition of $\eta_{1}$.

REMARK 4.2 .

1) We note that we can extend the definition of $\eta_{1}(t)$ for $t>0$ :

$$
\eta_{1}(t, \omega):=\eta_{1}\left(t-[t], \theta_{[t]} \omega\right) \in C\left([0, \infty) ; D\left(A^{\gamma}\right)\right)
$$

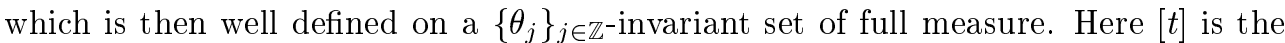
integer part of $t$. This process can be interpreted as a solution of (4.2) with initial condition $\eta_{1}(0, \omega)$.

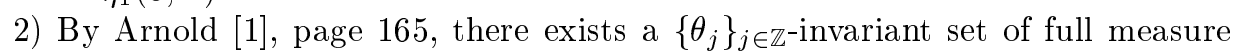
such that

$$
j \rightarrow \sup _{t \in[0,1]}\left\|\eta_{1}\left(t, \theta_{j} \omega\right)\right\|_{D\left(A^{\gamma}\right)}^{2}
$$

is subexponentially growing for $j \rightarrow \pm \infty$ : for any $\varepsilon>0$ there exists a $j_{0}$ such that

$$
\sup _{t \in[0,1]}\left\|\eta_{1}\left(t, \theta_{j} \omega\right)\right\|_{D\left(A^{\gamma}\right)}^{2} \leq e^{\varepsilon|j|}
$$

for $|j|>j_{0}$.

3) The constant $C\left(\operatorname{tr}_{U} Q\right)$ can be made arbitrarily small if $\operatorname{tr}_{U} Q$ is sufficiently small.

4) $\eta_{1}(t)$ is a Gaussian process. Hence we have automatically from 2 ) that

$$
\mathbb{E} \sup _{t \in[0,1]}\left\|\eta_{1}(t)\right\|_{D\left(A^{\gamma}\right)}^{n}<\infty \text { for any } n \in \mathbb{N} \text {. }
$$

In a similar manner we can consider (4.2) with time-periodic boundary condition representing the impact of the earth's rotation on the fluid:

$$
\eta_{2 t}=\nu \tilde{\Delta} \eta_{2}, \frac{\partial \eta_{2}}{\partial n}=0 \quad \text { on } \quad O_{,,, 0}, \quad \frac{\partial \eta_{2}}{\partial n}=u_{p} \sin (2 \pi t) \quad \text { on } \quad O_{,,, 2 \pi}
$$

for $u_{p} \in H^{\frac{1}{2}}\left(O_{,, 2 \pi}\right) \cap \dot{L}_{2}\left(O_{,, 2 \pi}\right)$ and (spatial) periodic boundary conditions on the other faces. We obtain without proof the following:

Lemma 4.3. Suppose that $u_{p} \in H^{\frac{1}{2}}\left(O_{,,, 2 \pi}\right) \cap \dot{L}_{2}\left(O_{,,, 2 \pi}\right)$. Then there exists a continuous time-periodic solution

$$
t \rightarrow \eta_{2}(t) \in H^{2} \subset D\left(A^{\rho}\right), \quad \rho<\frac{1}{4}
$$

which satisfies (4.4). In particular, $\eta_{2}(t)$ is also periodic in $x$ and $y$. The proof is based on the properties of the operator $G$. 
Let $\theta^{2}=\left(\theta^{2}\right)_{t \in \mathbb{R}}$ be the shift operator $\theta_{t}^{2} f(\cdot)=f(t+\cdot)$ for appropriate functions $f$. We consider the hull of $u_{p} \sin (2 \pi t)$ with respect to $\theta^{2}$ :

$$
\Omega_{2}=\bigcup_{t \in \mathbb{R}} \theta_{t}^{2}\left(u_{p} \sin (2 \pi \cdot)\right)=\bigcup_{t \in \mathbb{R}} u_{p} \sin (2 \pi(t+\cdot))=\bigcup_{t \in[0,2 \pi)} u_{p} \sin (2 \pi(t+\cdot)) .
$$

Summarizing, we have found a process $\eta=\eta_{1}+\eta_{2}$ which will serve as a model for the perturbation on the ocean surface.

After these preparations, we can introduce a nonautonomous/random dynamical system. Let $\theta=\left(\theta^{1}, \theta^{2}\right)$ be a flow on a set $\Omega=\Omega_{1} \times \Omega_{2}$ where $\theta^{1}$ is defined by (4.1) on $\Omega_{1}$ and $\theta^{2}$ describes the periodic motion on $\Omega_{2}$. Let $\mathbb{T}$ be $\mathbb{Z}$ or $\mathbb{R}$.

Definition 4.4. Let $(\Omega, \mathcal{F}, \mathbb{P}, \theta)$ be a metric dynamical system. A random dynamical dynamical system $\phi$ on a phase space $H$ with respect to $\theta$ is a measurable mapping

$$
\phi: \mathbb{T}^{+} \times \Omega \times H \rightarrow H
$$

fulfilling the cocycle property

$$
\begin{aligned}
\phi(t+\tau, \omega, \cdot) & =\phi\left(t, \theta_{\tau} \omega, \phi(\tau, \omega, \cdot)\right) \quad \text { for } t, \tau \in \mathbb{T}^{+}, \\
\phi(0, \omega, \cdot) & =\operatorname{id}_{H}
\end{aligned}
$$

for $\omega \in \Omega$ and $t, \tau \in \mathbb{T}^{+}$. This definition is due to Arnold [1].

REMARK 4.5. We will denote this solution operator of (3.1) by $\phi(t, \omega, x)$ which satisfies the properties of the above definition. Note that by the time-periodicity of $\eta_{2}$ the restriction of $\phi$ on $\mathbb{T}^{+}=\mathbb{Z}$ is a random dynamical system for any fixed $\omega_{2} \in \Omega_{2}$. Indeed, $\theta_{i} \omega=\left(\theta_{i}^{1} \omega_{1}, \omega_{2}\right)$ which can be identified with $\theta^{1}$ and which leaves $\mathbb{P}$ invariant $\left(\mathbb{P}\right.$ is ergodic with respect to $\left.\left(\theta_{i}^{1}\right)_{i \in \mathbb{Z}}\right)$.

Remark 4.6. Another opportunity to get an example for a complete random dynamical system would be to equip $\Omega_{2}$ with an ergodic measure. But in contrast to the fact that the daily or yearly rotation of the earth is well determined such a random ansatz would express that the beginning of these periods is rather random.

We now consider the existence of an attractor for the nonautonomous dynamical system. This attractor will attract random sets in probability. Before we give the theorem, we make some basic remarks on random sets.

Suppose that $H$ is a Polish space. A set function $\omega \rightarrow D(\omega)$ with closed and nonempty images is called a closed random set over $\left(\Omega_{1}, \mathcal{F}, \mathbb{P}\right)$ if and only if there exists a countable number of random variables

$$
\xi_{i}:\left(\Omega_{1}, \mathcal{F}, \mathbb{P}\right) \rightarrow H, \quad i \in \mathbb{N}
$$

such that

$$
D(\omega)=\overline{\bigcup_{i \in \mathbb{N}} \xi_{i}(\omega)}
$$

See Castaing and Valadier [5], Chapter 3.

A random set $D$ is called of subexponential growth if $j \rightarrow \operatorname{dist}_{H}\left(D\left(\theta_{j} \omega\right),\{0\}\right)$ is of subexponential growth for $j \rightarrow \pm \infty$ where

$$
\operatorname{dist}_{H}(A, B)=\sup _{a \in A} \inf _{b \in B}\|a-b\|_{H} .
$$


See [1] for more information on subexponential growth. In the following we will denote the set of all of these random sets $D$ with closed and nonempty images $D(\omega)$ by $\mathcal{D}$.

We now define the term random attractor.

DEFINITION 4.7. Let $\phi$ be a random dynamical system over the metric dynamical system $\theta$. A set $(A(\omega))_{\omega \in \Omega} \in \mathcal{D}$ with compact and nonempty images $A(\omega)$ is called random attractor if

$$
\phi(t, \omega, A(\omega))=A\left(\theta_{t} \omega\right), \quad t \in \mathbb{T}^{+}
$$

and for any set $D(\omega) \in \mathcal{D}$ :

$$
(\mathbb{P}) \lim _{t \rightarrow \infty, t \in \mathbb{T}^{+}} \operatorname{dist}_{H}\left(\overline{\phi(t, \omega, D(\omega))}, A\left(\theta_{t} \omega\right)\right)=0 .
$$

In the next section we will show that the dynamical system generated by (3.1) restricted to $\mathbb{T}=\mathbb{Z}$ has such a random attractor. However, because of time-periodic perturbation, we do not have a random dynamical system but a nonautonomous dynamical system for $\mathbb{T}=\mathbb{R}$. Therefore we have to modify this conclusion for $\mathbb{T}=\mathbb{Z}$ a little bit, and thus we obtain the following main result in this section.

THEOREM 4.8. Let $\phi(t, \omega, x)$ be the solution operator of (3.1) for the random and time-periodic forcing $\eta=\eta_{1}+\eta_{2}$. Suppose that

$$
\nu \lambda_{1}-8 c\left(\left\|u_{p}\right\|_{D\left(A^{\gamma}\right)}^{4}+\mathbb{E} \sup _{t \in[0,1]}\left\|\eta_{1}(t)\right\|_{D\left(A^{\gamma}\right)}^{4}\right)>0
$$

where $c$ is a constant depending on the data. Then the $3 D$ quasigeostrophic flow model (3.1) restricted to $\mathbb{T}^{+}=\mathbb{Z}^{+}$has a random attractor.

If we extend $A\left(\theta_{k}\left(\omega_{1}, \omega_{2}\right)\right)$ by $A\left(t-[t], \theta_{[t]}\left(\omega_{1}, \omega_{2}\right)\right):=\phi\left(t-[t], \theta_{[t]} \omega, A\left(\theta_{[t]}\left(\omega_{1}, \omega_{2}\right)\right)\right.$ for $t \in[0,1]$. Then (4.6) and (4.5) are also satisfied if we replace $A\left(\theta_{t}\left(\omega_{1}, \omega_{2}\right)\right)$ by $A\left(t-[t], \theta_{[t]}\left(\omega_{1}, \omega_{2}\right)\right)$ for $t \in \mathbb{R}^{+}$. In this way, the $3 D$ quasigeostrophic flow model (3.1) restricted to $\mathbb{T}^{+}=\mathbb{R}^{+}$has a random attractor.

The set valued process $(t, \omega) \rightarrow A\left(t-[t], \theta_{t}\left(\omega_{1}, \omega_{2}\right)\right)$ can be interpreted as a version of $t \rightarrow A\left(\theta_{t}\left(\omega_{1}, \omega_{2}\right)\right)$ which fits the Definition 4.7 for continuous time.

In the next section, we prove Theorem 4.8 .

\section{Proof of the Main Theorem}

The following theorem states the existence of random attractors.

THEOREM 5.1. Suppose that $\theta^{1}$ be a metric dynamical system and suppose that for $\omega_{1} \in \Omega_{1}, t \in \mathbb{Z}^{+}$the mappings $\phi\left(t, \omega_{1}, \cdot\right)$ are continuous and that there exists a set $B \in \mathcal{D}$ having compact images which is absorbing:

$$
\phi\left(t, \theta_{-t}^{1} \omega_{1}, D\left(\theta_{-t}^{1} \omega_{1}\right)\right) \subset B\left(\omega_{1}\right)
$$

for $\mathbb{Z}^{+} \ni t \geq t_{0}(D, \omega)$, and any $D \in \mathcal{D}$. Then there exists a unique random attractor $A \in \mathcal{D}$ which is tempered (Tempered random sets are defined in Arnold [1]).

The proof of this theorem can be found in Flandoli and Schmalfuß [11]. 
Now let us use this result to prove our main Theorem 4.8 .

Proof. We start with some energy estimates. Calculating the inner product in $H$ and using Lemma 3.6 and Lemma 3.9, we obtain

$$
\begin{aligned}
\|u(t)\|_{H}^{2} & +2 \nu \int_{0}^{t}\|u(\tau)\|_{V}^{2} d \tau=\left\|u_{0}\right\|_{H}^{2}-2 \beta \int_{0}^{t}\left\langle G(\eta)_{x}, u\right\rangle d \tau \\
& -2 \int_{0}^{t}\langle J(G(u), \eta), u\rangle d \tau-2 \int_{0}^{t}\langle J(G(\eta), \eta), u\rangle d \tau
\end{aligned}
$$

By Lemma 3.5 and the Young inequality, we get for some $c>0$

$$
2|\langle J(G(u), \eta), u\rangle| \leq \frac{\nu}{3}\|u\|_{V}^{2}+c\|\eta\|_{D\left(A^{\gamma}\right)}^{4}\|u\|_{H}^{2}
$$

and for the other terms on the right hand side, we further get

$$
\begin{aligned}
2|\langle J(G(\eta), \eta), u\rangle| & \leq \frac{\nu}{3}\|u\|_{V}^{2}+c\|\eta\|_{D\left(A^{\gamma}\right)}^{4} \\
2\left|\left\langle G(\eta)_{x}, u\right\rangle\right| & \leq \frac{\nu}{3}\|u\|_{V}^{2}+c\|\eta\|_{D\left(A^{\gamma}\right)}^{2}
\end{aligned}
$$

Since $\|u\|_{V}^{2} \geq \lambda_{1}\|u\|_{H}^{2}$ for $u \in V$

$$
\begin{aligned}
\|u(t)\|_{H}^{2} & +\nu \lambda_{1} \int_{0}^{t}\|u(\tau)\|_{H}^{2} d \tau \leq\left\|u_{0}\right\|_{H}^{2}+c \int_{0}^{t}\|\eta(\tau)\|_{D\left(A^{\gamma}\right)}^{4}\|u(\tau)\|_{H}^{2} d \tau \\
& +\int_{0}^{t} P\left(\|\eta(\tau)\|_{D\left(A^{\gamma}\right)}\right) d \tau
\end{aligned}
$$

$P$ is some appropriate polynomial of order four with $P(0)=0$ estimating the terms containing $\|\eta\|_{D\left(A^{\gamma}\right)}$. Parallel to this inequality, we consider the affine equation

$$
\frac{d \xi}{d t}+\left(\nu \lambda_{1}-c\|\eta(t)\|_{D\left(A^{\gamma}\right)}^{4}\right) \xi=P\left(\|\eta(t)\|_{D\left(A^{\gamma}\right)}\right), \quad \xi_{0} \geq\left\|u_{0}\right\|_{H}^{2}
$$

We now fix for a while $\omega_{2}=\bar{\omega}_{2}$. The solution of this equation $\xi\left(t, \omega,\left\|u_{0}\right\|_{H}^{2}\right)$ for $\omega=\left(\omega_{1}, \bar{\omega}_{2}\right)$ is a bound for $\|u(t)\|_{H}^{2}$ :

$$
\|u(t)\|_{H}^{2} \leq \xi\left(t, \omega,\left\|u_{0}\right\|_{H}^{2}\right) .
$$

We restrict the solution of $(3.1)$ to discrete times. Let $\xi^{*}(\omega)$ be the following random variable:

$$
\sum_{i=-\infty}^{0} e^{\nu \lambda_{1} i-c \sum_{j=-1}^{i-1} \sup _{t \in[0,1]}\left\|\eta\left(t, \theta_{j} \omega\right)\right\|_{D\left(A^{\gamma}\right)}^{4}} \sup _{t \in[0,1]} P\left(\left\|\eta\left(t, \theta_{i-1} \omega\right)\right\|_{D\left(A^{\gamma}\right)}\right)
$$

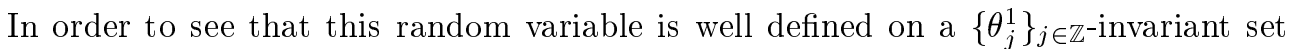
of full measure, we mention the exponent in this formulae growth to $-\infty$ with linear

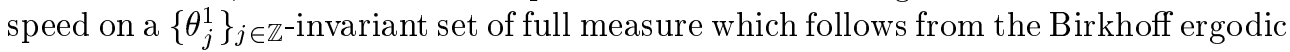


theorem and (4.7):

$$
\begin{aligned}
\limsup _{i \rightarrow \pm \infty} & \frac{1}{|i-1|} \sum_{j=-1}^{i-1}\left(\nu \lambda_{1}+c \sup _{t \in[0,1]}\left\|\eta\left(t, \theta_{j} \omega\right)\right\|_{D\left(A^{\gamma}\right)}^{4}\right) \\
& \leq \lim _{i \rightarrow \pm \infty} \frac{1}{|i-1|} \sum_{j=-1}^{i-1}\left(-\nu \lambda_{1}+8 c\left(\left\|u_{p}\right\|_{D\left(A^{\gamma}\right)}^{4}+\sup _{t \in[0,1]}\left\|\eta_{1}\left(t, \theta_{j} \omega\right)\right\|_{D\left(A^{\gamma}\right)}^{4}\right)\right) \\
& =-\nu \lambda_{1}+8 c\left(\left\|u_{p}\right\|_{D\left(A^{\gamma}\right)}^{4}+\mathbb{E} \sup _{t \in[0,1]}\left\|\eta_{1}(t)\right\|_{D\left(A^{\gamma}\right)}^{4}\right)<0
\end{aligned}
$$

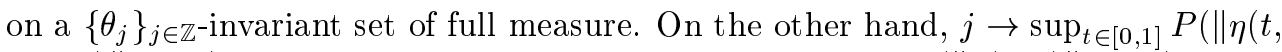
$\left.\left.\theta_{j-1} \omega\right) \|_{D\left(A^{\gamma}\right)}\right)$ has a subexponential growth since $\mathbb{E}_{\sup _{t \in[0,1]}} P\left(\|\eta(t, \omega)\|_{D\left(A^{\gamma}\right)}\right)$. Hence, the mapping $j \rightarrow \xi^{*}\left(\theta_{j} \omega\right)$ is subexponentially growing which follows from [28] Section 2 or [12].

A straightforward calculation shows $\xi\left(1, \omega, \xi^{*}(\omega)\right) \leq \xi^{*}\left(\theta_{1} \omega\right)$ and by iteration

$$
\xi\left(k, \omega, \xi^{*}(\omega)\right) \leq \xi^{*}\left(\theta_{k} \omega\right) .
$$

Since

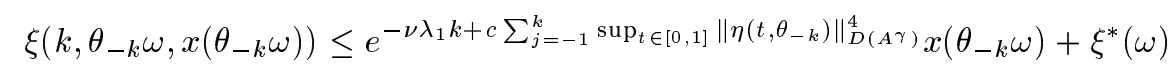

for sufficiently large $k \geq k_{0}(\omega, x)$ and if $j \rightarrow x\left(\theta_{j} \omega\right)$ is subexponentially growing for $j \rightarrow \pm \infty$, we have by $(5.5)$

$$
\lim _{k \rightarrow \infty} e^{-\nu \lambda_{1} k+c \sum_{j=-1}^{k} \sup _{t \in[0,1]}\left\|\eta\left(t, \theta_{-k}\right)\right\|_{D(A \gamma)}^{4}} x\left(\theta_{-k} \omega\right)=0 .
$$

According to this property, we can conclude

$$
\operatorname{dist}_{H}\left(\phi\left(k, \theta_{-k} \omega, D\left(\theta_{-k} \omega\right)\right), 0\right) \leq 2 \xi^{*}(\omega)
$$

for $D \in \mathcal{D}$ and sufficiently large $k$. Similarly we have for large $k$

$$
\operatorname{dist}_{H}(\phi(k, \omega, D(\omega)), 0) \leq 2 \xi^{*}\left(\theta_{k} \omega\right) .
$$

We can conclude that the closed ball $\tilde{B}(\omega)$ in $H$ with center zero and radius $2 \xi^{*}(\omega)$ is a forward and backward absorbing (see (5.1)) set and is also forward invariant (see $5.6)$ :

$$
\phi(k, \omega, \tilde{B}(\omega)) \subset \tilde{B}\left(\theta_{k} \omega\right) .
$$

On the other hand, we note that

$$
B(\omega):=\overline{\phi\left(1, \theta_{-1} \omega, \tilde{B}\left(\theta_{-1} \omega\right)\right)} \subset \tilde{B}(\omega)
$$

is compact, because $\phi$ is regularizing for $t>0$ which follows from Lemma 3.12. In addition, this set is a random set since $\phi(t, \omega, \cdot)$ is continuous which follows from Lemma 3.11 Moreover, $B$ is absorbing in the sense of (5.1) which follows by the definition of $B(\omega)$ and the cocycle property.

Theorem 5.1 ensures the existence of a random attractor stated in the first part of Theorem 4.8. 
We show convergence

$$
(\mathbb{P}) \lim _{t \rightarrow \infty, t \in \mathbb{R}^{+}} \operatorname{dist}_{H}\left(\overline{\phi\left(t,\left(\omega_{1}, \bar{\omega}_{2}\right), D\left(\left(\omega_{1}, \bar{\omega}_{2}\right)\right)\right)}, A\left(t-[t], \theta_{[t]}\left(\omega_{1}, \bar{\omega}_{2}\right)\right)\right)=0
$$

for $D \in \mathcal{D}$ and any $\bar{\omega}_{2} \in \Omega_{2}$. Since $B(\omega)$ is a forward absorbing and forward invariant set for any $D \in \mathcal{D}$, it remains to check the convergence conclusion for $D=B$. On account of (5.2) and (5.3), we notice that for fixed $\bar{\omega}_{2}$ and $u_{1}=\phi\left(t,\left(\omega_{1}, \bar{\omega}_{2}\right), y\right)$,

$$
\begin{aligned}
\sup _{y \in B\left(\left(\omega_{1}, \bar{\omega}_{2}\right)\right)} \nu & \int_{0}^{1}\left\|\phi\left(\tau,\left(\omega_{1}, \bar{\omega}_{2}\right), y\right)\right\|_{V}^{2} d \tau \leq 2 \xi^{*}\left(\omega_{1}, \bar{\omega}_{2}\right) \\
& +\int_{0}^{1} P\left(\|\eta(\tau)\|_{D\left(A^{\gamma}\right)}\right)+c\|\eta(\tau)\|_{D\left(A^{\gamma}\right)}^{4} \xi\left(\tau,\left(\omega_{1}, \bar{\omega}_{2}\right), \xi^{*}\left(\left(\omega_{1}, \bar{\omega}_{2}\right)\right)\right) d \tau .
\end{aligned}
$$

We obtain by (3.2) an estimate of $\left\|u_{1}(t)-u_{2}(t)\right\|_{H}^{2}$ if we know the difference of $\left\|u_{1}([t])-u_{2}([t])\right\|_{H}^{2}$ such that

$$
\left\|u_{1}(t)-u_{2}(t)\right\|_{H}^{2} \leq Y\left(\left(\omega_{1}, \omega_{2}\right)\right)\left\|u_{1}([t])-u_{2}([t])\right\|_{H}^{2} .
$$

The random variable $Y$ can be derived from the variation of constants formula from (3.2)

$$
\begin{aligned}
& \sup _{y \in B\left(\left(\omega_{1}, \bar{\omega}_{2}\right)\right)} e^{\tilde{c} \int_{0}^{1}\left\|\phi\left(\tau,\left(\omega_{1}, \bar{\omega}_{2}\right), y\right)\right\|_{V}^{2} d \tau+\hat{c} \sup _{t \in[0,1]}\|\eta(t)\|_{D(A \gamma)}^{4}} \\
& \leq \exp \left(\bar{c}\left(2 \xi^{*}\left(\omega_{1}, \bar{\omega}_{2}\right)\right)+\int_{0}^{1} P\left(\|\eta(\tau)\|_{D\left(A^{\gamma}\right)}\right)\right. \\
& \left.+2 c\|\eta(\tau)\|_{D\left(A^{\gamma}\right)}^{4} \xi\left(\tau,\left(\omega_{1}, \bar{\omega}_{2}\right), \xi^{*}\left(\left(\omega_{1}, \bar{\omega}_{2}\right)\right)\right) d \tau+\hat{c} \sup _{t \in[0,1]}\|\eta(t)\|_{D\left(A^{\gamma}\right)}^{4}\right)=: Y\left(\left(\omega_{1}, \bar{\omega}_{2}\right)\right)
\end{aligned}
$$

for appropriate constants $\tilde{c}, \hat{c}, \bar{c}$, and $c$. The mapping

$$
\left(\omega_{1}, n\right) \rightarrow Y\left(\left(\theta_{n}^{1} \omega_{1}, \bar{\omega}_{2}\right)\right)=Y\left(\theta_{n}\left(\omega_{1}, \bar{\omega}_{2}\right)\right)
$$

defines a stationary process. On account of this inequality, we can conclude that

$$
\begin{aligned}
& \sup _{\tau \in[0,1]} \operatorname{dist}_{H}\left(\phi\left(\tau+n, \omega, B\left(\left(\omega_{1}, \bar{\omega}_{2}\right)\right)\right), A\left(\tau, \theta_{n}\left(\omega_{1}, \bar{\omega}_{2}\right)\right)\right) \\
& \quad \leq \operatorname{dist}_{H}\left(\phi\left(n,\left(\omega_{1}, \bar{\omega}_{2}\right), B\left(\left(\omega_{1}, \bar{\omega}_{2}\right)\right)\right), A\left(\left(\theta_{n}^{1} \omega_{1}, \bar{\omega}_{2}\right)\right)\right) Y\left(\left(\theta_{n}^{1} \omega_{1}, \bar{\omega}_{2}\right)\right)^{\frac{1}{2}} .
\end{aligned}
$$

Since the first factor of the right-hand side tends to zero in probability, the product of the right-hand side also tends to zero in probability. This gives the convergence conclusion (4.6). The proof of Theorem 4.8 is complete.

\section{Summary}

We have studied the 3D baroclinic quasigeostrophic flow model under random wind forcing and time-periodic fluctuations on fluid boundary; i.e., on the air-sea interface. The time-periodic fluctuations are due to periodic rotation of the earth and thus periodic exposure of the earth to the solar radiation. We have established the well-posedness of the baroclinic quasigeostrophic flow model in the state space (Theorem 3.10), and we have demonstrated the existence of the random attractors (Theorem 4.8), again in the state space. We have also discussed the relevance of our results to climate modeling. 
Acknowledgement. A part of this work was done at the Oberwolfach Mathematical Research Institute, Germany, supported by Volkswagen Stiftung, while the authors were Research in Pairs Fellows. This work was also partly supported by the NSF Grant DMS-0139073.

\section{REFERENCES}

[1] L. Arnold, Random Dynamical Systems. Springer, New York, 1998.

[2] L. Arnold, Hasselmann's program visited: The analysis of stochasticity in deterministic climate models. Report 450, Universität Bremen, Institut für Dynamische Systeme, 2000.

[3] A.J. Bourgeois and J.T. Beale, Validity of the quasigeostrophic model for large-scale flow in the atmosphere and ocean. SIAM J. Math. Anal., 25:1023-1068, 1994.

[4] J.R. Brannan, J. Duan, and T. Wanner, Dissipative quasigeostrophic dynamics under random forcing. J. Math. Anal. Appl., 228:221-233, 1998.

[5] C. Castaing and M. Valadier, Convex Analysis and Measurable Multifunctions. LNM 580. Springer-Verlag, Berlin-Heidelberg-New York, 1977.

[6] B. Desjardins and E. Grenier, Derivation of quasigeostrophic potential vorticity equations. Adv. Diff. Eqns. 3:715-752, 1998.

[7] A.F. Bennett and P.E. Kloeden, The dissipative quasigeostrophic equations. Mathematika, 18:265-288, 1981

[8] J. Duan, A remark on three-dimensional baroclinic quasigeostrophic dynamics. Appl. Math. and Comput., 106:285-289, 1999.

[9] Y.V. Egorov and M.A. Shubin, Partial Differential Equations. Volume I of Encyclopaedia of Mathematical Sciences. Springer, New York, 1991.

[10] P.F. Embid and A.J. Majda, Averaging over fast gravity waves for geophysical flows with arbitrary potential vorticity. Comm. PDEs, 21:619-658, 1996.

[11] F. Flandoli and B. Schmalfuß, Random attractors for the stochastic 3-D Navier-Stokes equation with multiplicative white noise. Stochatics and Stochastics Reports, 59:21-45, 1996.

[12] F. Flandoli and B. Schmalfuß, Weak solutions and attractors for the $3 D$ Navier-Stokes equation with nonregular force. J. Dynamics and Differential Equations, 11:355-397, 1999.

[13] A.V. Fursikov, Optimal Control of Distributed Systems-Theory and Applications. Amer. Math. Society, Providence, Rhode Island, 2000.

[14] K. Hasselmann, Stochastic climate models, part I. Tellus, 28:473-485, 1976.

[15] G. Holloway, Ocean Circulation: Flow in Probability Under Statistical Dynamical Forcing. In Stochastic Models in Geosystems, S. Molchanov and W. Woyczynski (eds.), Springer, 1996.

[16] D.D. Holm, Hamiltonian formulation of the baroclinic quasigeostrophic fluid equations. Phys. Fluids, 29:7-8, 1986.

[17] M. Leroux, Dynamic Analysis of Weather and Climate. John Wiley \& Sons, 1998.

[18] P. Müller, Stochastic Forcing of Quasigeostrophic Eddies. In Stochastic Modelling in Physical Oceanography, P. Müller R. J. Adler and B. Rozovskii, (eds.), 381-396. Birkhäuser, Basel, 1996.

[19] C. Nicolis and G. Nicolis, Is there a climate attractor? Nature, 311, 1984.

[20] G.R. North and R.F. Cahalan, Predictability in a solvable stochastic climate model. J. Atmos. Sci., 38:504-513, 1981.

[21] J. Pedlosky, Geophysical Fluid Dynamics. Springer Verlag, New-York, Berlin, 1987.

[22] J.P. Peixoto and A.H. Oort, Physics of Climate. Springer, New York, 1992.

[23] G. Da Prato and J. Zabczyk, Stochastic Equations in Infinite Dimension. Combridge University Press, Cambridge, 1992.

[24] G. Da Prato and J. Zabczyk, Evolution equations with white noise boundary conditions. Stochastics and Stochastics Reports, 42:167-182, 1993.

[25] G. Da Prato and J. Zabczyk, Ergodicity for Infinite Dimensional Systems. Cambridge University Press, Cambridge, 1996.

[26] R. Salmon, Lectures on Geophysical Fluid Dynamics. Oxford University Press, Oxford, 1998.

[27] R.M. Samelson, Stochastically forced current fluctuations in vertical shear and over topography. J. Geophys. Res. 94:8207-8215, 1989.

[28] B. Schmalfuß, A random fixed point theorem and the random graph transformation. J. Math. Anal. Appl., 225:91-113, 1998.

[29] R. Temam, Navier-Stokes Equation-Theory and Numerical Analysis. North-Holland, Amsterdam, 1979. 
[30] R. Temam, Navier-Stokes Equation and Nonlinear Functional Analysis. CBMS-NSF Regional Conference Series in Applied Mathematics, SIAM, Philadelphia, 1983.

[31] R. Temam, Infinite-Dimensional Dynamical Systems in Mechanics and Physics. SpringerVerlag, Berlin-Heidelberg-New York, second edition, 1997.

[32] S. Wang, Attractors for the 3D baroclinic quasigeostrophic equations of large-scale atmosphere. J. Math. Anal. Appl., 165:266-282, 1992. 